\title{
EDITORIAL
}

\section{HOPING FOR THE MOTHER OF ALL MERGERS}

\author{
As another pharmaceutical company merger comes to completion, the greatest challenge \\ remains the integration of the academic and industrial research cultures.
}

Unless we're all to be greatly surprised, by the time this issue reaches readers the birth of a new French pharmaceutical giant will have been confirmed by the shareholders, and Sanofi-Aventis - if that is to be its name will have come into existence. All the 'will they, won't they' speculation will be over, and the integration teams charged with the task of making the most of this combination might already be at work. Their greatest challenge, at least on the science side, will be the integration of the different research cultures within the two organizations.

So it is with all mergers. Each institution has its own way of doing things, and company heritage is a strong lineage marker. Those working in pharmaceutical companies formed from long sequences of multiple mergers will certainly be able to tell you of colleagues who still identify themselves very strongly as belonging to one or other of the predecessor companies. Although their original homes are now long gone, SmithKline Beecham and Sandoz (to pick two names at random from any number of possible heritages) researchers are still very much alive. Of course, it is right and proper that people should be proud of their companies and wedded to the research culture they found, or indeed helped to found, there. But finding ways to best exploit such loyalty to the 'old ways' within a new framework is also one of the challenges facing every merged entity, and so such companies rightly, and increasingly, put great effort into developing formulae to ease the transition.

Concerns about the cultural mix of scientists in newly merged pharmaceutical companies, however, can seem child's play compared with the perennial mother of all integration challenges: bridging the industrial-academic divide. Once again, the fact that these different approaches to biomedical problems flourish - the one so very applied, the other so ultimately free of all utility obviously adds welcome diversity to drug research. Needless to say, the benefits of combining such approaches should be great. But in this case, the integration teams' are non-existent, and relatively little effort is expended on forcing the two camps to engage with each other. Researchers are still, in the main, labelled as belonging to one or the other camp, and the worrying thing is that both camps are content with this modus vivendi of labelling each other as being somehow 'different'.

During some of the discussions at the recent Horizon Symposium on 'Charting chemical space', the ease with which people can fall naturally into making these distinctions was neatly highlighted (see www.horizonsymposia.com for highlights of the meeting). For example, in one session that focused on the improvement of biological assays, delegates from industry admitted that their primary assays used to screen for biologically active compounds, or 'hits', are 'dirty', with results often differing significantly depending on the assay used. But broadly speaking, those from industry seemed content with this situation; in their view, the main use for these assays is as a rapid, cost-effective sieve to identify potential chemical starting points for the development of lead series that will be much more thoroughly characterized. However, academic delegates in general advocated the development of more informative - but also more time-consuming and costly - assays with a much greater emphasis on data quality, with a view to creating large databases correlating chemical structure with biological function that they suggest could prove invaluable for a better understanding of biological processes. Both approaches had strong proponents and opponents, but the interesting phenomenon was how readily these positions were perceived as either 'industrial' or 'academic', and therefore more easily dismissible by the other group.

Arguing strongly for the case that academic-industrial integration is not only desirable but actually a prerequisite for industrial success is the fact that those based in universities, biotechs and pharmaceutical companies alike are ever-increasingly recognizing the frightening complexity of disease biology. Merging the expertise provided by these different approaches to biomedical research is the greatest challenge we face. 